\title{
On a finite domain magnetic localization by means of TMR triaxial sensors
}

\author{
G. Cerro, L. Ferrigno, M. Laracca, F. Milano \\ Dept. Electrical and Information Engineering \\ University of Cassino and Southern Lazio \\ Cassino, Italy \\ \{g.cerro, ferrigno, m.laracca, filippo.milano\}@unicas.it \\ O. Casas Piedrafita \\ Instrumentation, Sensors and Interfaces Group \\ Universitat Politècnica de Catalunya-BarcelonaTech \\ Castelldefels, Barcelona, Spain \\ \{Jaime.Oscar.Casas\}@upc.edu
}

\author{
P. Bellitti, M. Serpelloni \\ Department of Information Engineering \\ University of Brescia \\ Brescia, Italy \\ \{p.bellitti, mauro.serpelloni\}@unibs.it
}

\begin{abstract}
The paper proposes the realization and the preliminary characterization of a short-range localization systems based on the measurement of magnetic fields. Fixed anchors that generate sinusoidal wave magnetic fields at different frequencies and mobile anchors that measure the received magnetic field values compose the system. Main topics faced in the paper are: the realization and the preliminary characterization of threedimensional magnetic field sensors based on the suitable arrangement of single axis TMR magnetic sensors and the proposal of a novel, scalable and flexible localization set-up allowing multiple mobile agents without increasing the complexity and the operational time of the system. Thanks to the low cost and the flexibility of the proposal, the realized system has a wide impact on the possible applications in industrial, medical and smart-life fields. For example, it can be adopted in localization of probes that execute the defect detection in specimen, in the realization of low cost devices that help in recognizing the health-state of peoples affected by motor problems related to neurological diseases, in application related to the virtual reality, etc. At this stage, the obtained results place the measurement accuracy lower than $1 \mathrm{~cm}$ when the localization range is a cube of $30 \mathrm{~cm}$.

Index Terms-localization, magnetic measurement, magnetic sensors, calibration, signal processing, experimental characterization
\end{abstract}

\section{INTRODUCTION}

Nowadays, many applications related to industrial, medical and smart city sectors, require the use of mobile nodes that act as sensors or actuators and operate in tight spaces [1][5]. Among industrial applications [6], [7], non-destructive investigation techniques aimed at finding defects in materials through mobile probes fall into this scenario [8]-[11]. As for medical applications, monitoring of patients suffering from neurodegenerative diseases, such as Parkinson's disease, for which it is necessary to monitor the tremor of a hand rather than the posture of a patient in simple gymnastic exercises is actually performed by mobile wearable sensors [12]. In these applications, it is often also very important to know in real time the position of these mobile sensors so that the data they produce can be correlated to the motion data or to specific sensor positions in the reference scenario. For these reasons, an issue related to the development of short-range tracking systems arises. Typically, these systems cannot be developed adopting the usual GPS-based localization technologies or radio signals because indoor scenarios, the required accuracy and costs become insurmountable obstacles for these technologies. For these reasons, we are witnessing a rapid development of localization methods and systems explicitly designed for small-scale applications. The localization systems can be divided into two categories. The anchor-free that do not require references in fixed and known positions, and anchorbased ones that use a system of anchors with respect to which to calculate the positions of the sensors in the threedimensional space. When the purpose of localization is the identification of the speed, orientation or acceleration of sensor node within reference scenario, measurement systems based on inertial platforms are often used, when the absolute position of the sensor node it is crucial information for the application to be met, resorting to systems with fixed anchors. Although anchor-based systems offer the disadvantage of having to build a special reference infrastructure, they guarantee better accuracy, greater reliability and lower computational burden compared to anchor-free systems. Within the family of anchorbased techniques, short-range localization systems based on magnetic field measurements are recently being developed [11]. Even if these systems have the disadvantage of typically operating very small areas, less than one cubic meter, on the other hand, they are cheap, scalable and easily transportable, so as to potentially present themselves as systems of great diffusion. In this context, the authors participate in a national interest research project that aims at developing a scalable sixdegree of freedom short range tracking systems. They have developed, in collaboration with the University of Perugia, an anchor-based short-range localization system based on a mobile node equipped with a transmitting coil and anchor nodes, in a fixed and known position, which act as magnetic field measurement systems [13]-[15]. The transmitting coil and the 
receiving coils are tuned in resonance to maximize the efficiency in the generation and the sensitivity in the measurement of the magnetic field. Although the realized system has shown excellent behaviors in terms of measurement accuracy, it could present limitations as transmitting nodes number grows. Since the receiving coils are tuned in resonance with the transmitting node, it would be necessary to operate in time division when the transmitting nodes is more than one. This could limit the maximum number of mobile nodes simultaneously present in the considered scenario or the number of measurements per second. In addition, since the mobile node is a transmitting node, the demand for power required to guarantee a reasonable coverage radius could limit the battery life of the mobile nodes. For this reason, in this paper, stemming from the authors' experience in magnetic sensor characterization [16], planar domain localization [17], development of electronic devices [18], [19], processing of digital signals [20]-[22] propose the development and the first experimental characterization of a new short-range magnetic localization system based on the use of mobile nodes equipped with Tunnel Magneto Resistor (TMR) type magnetic field sensors. In the new system the anchors, placed in a fixed and known positions, use resonantly tuned coils to generate magnetic fields at different frequencies. The mobile nodes, each one equipped with a three-axis magnetic field sensor, receive the different magnetic fields generated by the transmitters and reconstruct the position of the node through appropriate algorithms in the frequency domain. Following the work, firstly a brief theoretical description of the operating principles of the two considered localization systems is presented. After that, the new proposed localization system is detailed. Subsequently, after a first characterization of the metrological performance, the measurement performance are evaluated in comparison to the previous system.

\section{THEORETICAL PRINCIPLES OF MAGNETIC LOCALIZATION SYSTEMS}

Magnetic localization systems consist of a combination of beacons, located in fixed and known positions, and mobile nodes, free to move in a limited domain. The goal of these systems is the determination of mobile nodes' position and orientation, by generating and measuring magnetic fields. In this work, a comparison between two magnetic localization systems with different architectures is provided. In the first system, the generation and measurement of alternating magnetic fields are in charge of circular planar coils. Transmitter nodes $(t x)$ are associated with the mobile nodes while receiver devices $(r x)$ are associated with the beacons. This system will be identified, hereinafter in the paper as a "coil system". The second system (the new proposed one), involves the generation of alternating magnetic fields through $t x$ coils and a magnetic field sensor, namely Tunnel Magneto-Resistance (TMR), is responsible for the receiving phase. Unlike the previous one, in this case the $t x$ coils represent the beacons, while the $r x$ devices act as mobile nodes, thus reversing the working principle with respect to the coil system. The second system examined will be called "sensor system" in the following of the paper. In both cases, the position and orientation of a mobile node is defined as a six-dimensional $\theta$ variable, $\left(\theta=\left(x, y, z, u_{x}, u_{y}, u_{z}\right)\right)$, where $(\mathrm{x}, \mathrm{y}, \mathrm{z})$ are the position coordinates in a Cartesian reference system, while $\left(u_{x}, u_{y}, u_{z}\right)$ are the unit vector representing the orientation of the mobile node with respect to the same Cartesian Coordinate System. The estimation of $\theta$ is carried out by means of a numerical technique. In particular, a minimization of a cost function (1) is used, defined on the basis of the difference between the theoretical and its corresponding experimental model.

$$
F(\theta)=\sum_{i=1}^{N_{r x}}\left[\tilde{G}_{i}-G_{i}(\theta)\right]^{2}
$$

In (1), $\tilde{G}_{i}$ and $G_{i}$ represent the measured and theoretical quantities, respectively. The "simplex" optimization algorithm [23] is used to minimize the cost function. For both systems, the "coil" and the "sensor" ones, the theoretical model used in order to estimate the magnetic field in a given position, is based on the magnetic dipole moment; according to this, it is possible to calculate the magnetic field vector $\vec{B}$ generated by $t x$ coil in an assigned point $\mathrm{P}(\mathrm{x}, \mathrm{y}, \mathrm{z})$ by eqn. (2).

$$
\vec{B}(P)=\frac{\mu_{0}}{4 \pi} \frac{m_{t x}}{d^{3}}\left[3\left(\hat{n}_{t x} \cdot \hat{n}_{d}\right) \hat{n}_{d}-\hat{n}_{t x}\right]
$$

In (2), $\mu_{0}$ is the vacuum permeability, $m_{t x}$ e $n_{t x}$ represent the length and direction of the magnetic dipole moment vector representative of the $t x$ coil, while $d$ e $\hat{n}_{d}$ identify the distance vector between the $t x$ coil and P. For the sensor system, $\vec{B}(P)$ represents the model term to be included in the cost function. The computation of $\vec{B}(P)$ is performed for each $t x$ coil and refers to the position assumed by the sensor. As regards the coil system, we exploit the phenomenon of electromagnetic induction on the receiving coils and use the same magnetic dipole moment theory to formalize theoretical induced voltage on each coil. To do this, we used eqn. (3):

$$
V_{i}=2 \pi f_{0} N_{i} S_{i} K_{i} \vec{B}_{i} \cdot \hat{n}_{i}
$$

where $f_{0}$ is the supply frequency of the $t x$ coil, $N_{i}$ e $S_{i}$ are the numbers of turns and the section of the $i_{t h} r x$ coil, $K_{i}$ is a gain term for the $i_{t h} r x$ coil, $\vec{B}_{i}$ is the magnetic field vector induced on $i_{t h}$ receiver position, and $\hat{n}_{i}$ is the space orientation of the $i_{t h}$ coil. Regarding the measured quantities, from one side (coil system) the induced voltages measured on each $r x$ coil are used, while for the sensor system the magnetic field vector measured on the three axes by the sensor is directly adopted.

\section{EXPERIMENTAL SET-UP}

\section{A. Coil system}

The coil system (see Fig. 1) consists of $17 r x$ coils, and a $t x$ coil; the geometric characteristics of the coils are shown in Table I.

All devices, $t x$ and $r x$ coils, represent $L C$ resonant circuits obtained connecting capacitors in parallel to the coils to obtain 


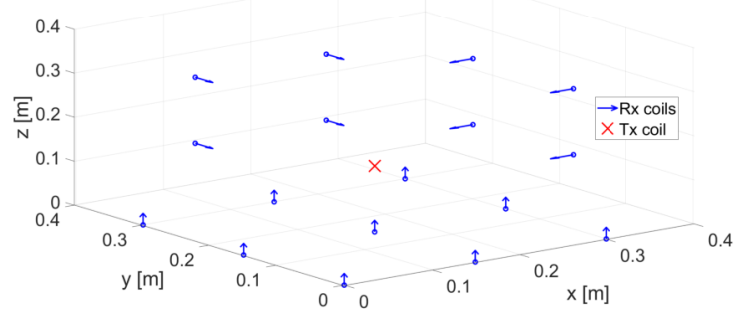

Fig. 1. Cartesian scheme of the coil system.

TABLE I

GEOMETRICAL FEATURES OF THE COILS IN THE DEVELOPED SYSTEM.

\begin{tabular}{lcr}
\hline \hline Main Features & tx coil & rx coil \\
\hline Radius & $5 \mathrm{~mm}$ & $20 \mathrm{~mm}$ \\
Height & $2 \mathrm{~mm}$ & $4 \mathrm{~mm}$ \\
Number of Turns & 15 & 5 \\
\hline
\end{tabular}

a resonant frequency of about $200 \mathrm{kHz}$. The $t x$ coil was driven by a HP $33120 \mathrm{~A}^{\mathrm{TM}}$ waveform generator, with a sinusoidal voltage signal with a frequency and voltage equal to $200 \mathrm{kHz}$ and $10 \mathrm{~V}$ peak-to-peak $(\mathrm{Vpp})$ respectively.

The induced voltage on the receiving coils was amplified by an Analog Device AD8421 ${ }^{\mathrm{TM}}$ instrumentation amplifier (INA), configured with a gain of 300 and supplied by an AGILENT E3631 $\mathrm{A}^{\mathrm{TM}}$ power supply with a voltage of $\pm 9 \mathrm{~V}$. The output of the INA was connected to a KEYSIGHT U2331A data acquisition unit.

\section{B. Sensor system}

The sensor system (depicted in Fig. 2) is composed of 24 resonant $t x$ coils placed in known and fixed positions and transmitting in frequency division mode and one receiving triaxial TMR sensor, composed by 3 single-axis sensors. The TMR is a magneto-resistive sensor based on the tunnel effect, i.e. the passage of electrons from one conductive layer to another occurs through the central insulating layer. The simplicity of tunneling between the two magnetic levels is modulated by the angle between the magnetization vectors in the two conductive layers. The presence of a magnetic field along the direction of sensitivity changes the angle between the magnetization vectors, causing a more or less intense passage of electrons through the insulating layer. The adopted sensor is the TMR2905D, which uses a single pushpull Wheatstone bridge composed of four unshielded TMR sensors. The unique bridge design provides a high sensitivity differential output linearly proportional with the magnetic field applied parallel to the package surface. The main features are: high sensitivity, wide dynamic range, excellent thermal stability and low hysteresis. From a physical point of view, the TMR2905D device has dimensions of $2.5 \mathrm{~mm} \times 2.5 \mathrm{~mm}$ $\mathrm{x} 0.9 \mathrm{~mm}$. It is able to receive data in a large frequency spectrum interval, thus allowing to exploit frequency diversity

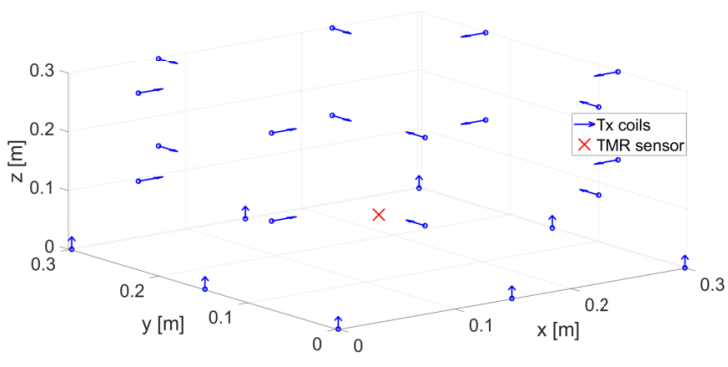

Fig. 2. Cartesian scheme of the sensor system.

in a system having more than one transmitter simultaneously generating magnetic field.

Two main electronic boards have been developed. The first one (XYZ board) used as a support to allow a triaxial arrangement of the TMR sensors and a second one (analog board) with the conditioning electronics and power supply circuit. The two boards can be connected using standard 2.54 $\mathrm{mm}$ header connector. XYZ board, reported in Fig. 3, has been designed, as well as a physical support, also as a breakout board allowing an easy connection of each pin of the devices mounted. In this way it is compatible even with conditioning method that requires access to each VCC and GND pin. (e.g. digital direct interface [24]). With reference to the origin shown in Fig. 3 the TMR sensor centroid are for X, Y and Zsensing device in $(4.5,9.6,0),(10,9.6,0),(14.19 .6$ 8.9) $\mathrm{mm}$. XYZ board dimensions are $18 \mathrm{~mm} \times 19.2 \mathrm{~mm}$. The second PCB board (Fig. 4) includes all the electronics to supply and perform signal conditioning of the TMR sensors output.

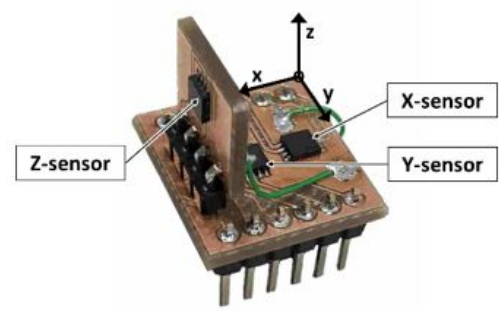

Fig. 3. XYZ board with soldered TMR2905 sensors and axes orientation.

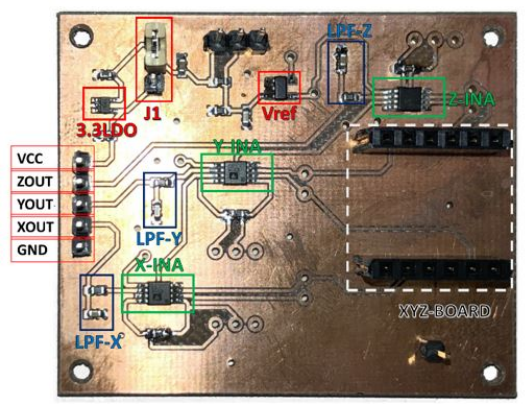

Fig. 4. Analog board with main blocks highlighted. 
The power supply block is composed by a TPS71533 LDO linear regulator (Texas Instrument) that provides a stable voltage of $3.3 \mathrm{~V}$ with an input range from $3.7 \mathrm{~V}$ to $24 \mathrm{~V}$, a jumper has been added to eventually exclude the regulator and apply an external voltage source. A low power operational amplifier MPC6001 (Microchip) provides a reference voltage of $\mathrm{VCC} / 2$ required for single supplied instrumentation amplifier (INA). INAs block are used to condition the differential signal provided by TMR sensors. Every sensor has its dedicated AD8223 (Analog Devices) INA connected to the sensor bridge output (V+ and V-). INAs gain has been set to 102.56 using an $820 \Omega$ resistor, additional place for a trimmer has been set up to allow fine gain tuning. At the output stage of each amplifier a first order passive low pass filter (LPF) has been placed. Finally, two row of female $2.54 \mathrm{~mm}$ header connector allow hosting the XYZ board. Analog board size: $58 \mathrm{~mm} \mathrm{x}$ $48 \mathrm{~mm}$.

In this preliminary characterization, the frequency diversity is applied also in time division mode. In detail, we divided the 24 coils in six groups, each one including four coils operating at different frequencies. Due to the current static context, each group is activated in a particular time slot in a serialized mode. Finally, when all the groups have been activated, the measured magnetic fields are gathered together and jointly analyzed. The transmission frequencies within each group are 26, 28, 36 and $40 \mathrm{kHz}$. Fig. 5 shows an example of measured signals when the TMR is in a known location. All the $t x$ coils are driven by an HP 33120A waveform generator with a voltage of $10 \mathrm{Vpp}$. The INA output was connected to a NI 6212 data acquisition unit.
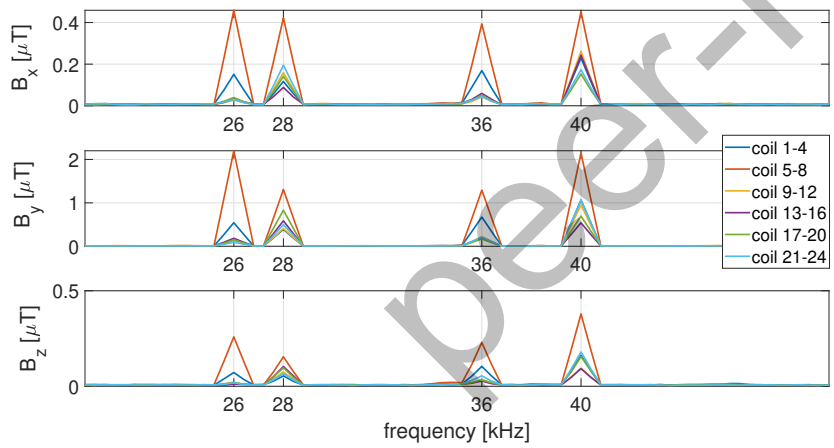

Fig. 5. Acquired signals for all $t x$ coils operating in time and frequency division.

\section{RESULTS}

\section{A. Sensor system: preliminary TMR characterization}

Before deploying the system for localization purposes, a preliminary characterization of the adopted TMR is needed because the factory data-sheet does not provide any information about its behavior in frequency domain. The characterization is therefore necessary to evaluate the measurement capabilities of the sensor and compensate unwanted effects. For this reason linearity, frequency stability, and sensitivity

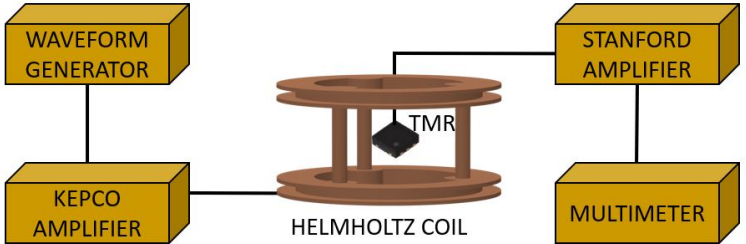

Fig. 6. Adopted set-up for the TMR characterization.

have been analyzed by adopting, as reference magnetic field generator, a pre-characterized Helmholtz coil. In particular the characterization is shown for a single TMR. Fig. 6 shows the adopted measurement set-up to characterize the TMR sensor. The sensor was placed in the middle of the Helmholtz coil, in order to be subjected to a uniform field. The Helmholtz coil was powered by HP $33120 \mathrm{~A}^{\mathrm{TM}}$ waveform generator and a KEPCO 2020 bipolar amplifier in order to generate a controlled magnetic field. Finally, the sensor output signal was amplified, using a STANFORD amplifier with a known gain, and measured with a multimeter. The tests were carried out by applying $\mathrm{AC}$ magnetic fields in the range from 0 to $4 \mathrm{G}$, with frequency values from $1 \mathrm{kHz}$ to $50 \mathrm{kHz}$. In all tests, the sensor was powered with a voltage equal to $1 \mathrm{~V}$. Fig. 7 shows the input-output characteristics for all analyzed frequencies. A good linearity was obtained, as shown by the $\mathrm{R}^{2}$ coefficients obtained through a linear fitting and shown in Table II. Furthermore, sensitivity values close to $40 \mathrm{mV} / \mathrm{V} / \mathrm{G}$ were obtained.

For the frequency stability test (shown in Fig. 8), we focus our attention in a narrower frequency interval, corresponding to the spectrum portion where the transmitting frequencies are located for the sensor system. In particular, we linearly spaced the frequency values inside the interval $[20,40] \mathrm{kHz}$. We evaluated the stability of the TMR frequency response by normalizing its output at the different frequency values with the response obtained at the central value of such interval according to the equation (4):

$$
\epsilon_{r}[\%]=\frac{B_{f_{i}}-B_{30}}{B_{30}} * 100
$$

where $B_{f_{i}}$ and $B_{30}$ are the TMR measured magnetic field at frequency $f_{i}$ and $30 \mathrm{kHz}$, respectively.

A current of $100 \mathrm{~mA}$ was given to the Helmholtz coil, obtaining a loss of stability from the sensor less than $0.6 \%$ in the frequency range of interest.

TABLE II

COEFFICIENTS OF DETERMINATION AND SENSITIVITY FOR THE ADOPTED TMR IN THE CONSIDERED FREQUENCY RANGE.

\begin{tabular}{ccc}
\hline \hline Frequency [kHz] & $\mathbf{R}^{\mathbf{2}}$ & Sensitivity [mV/V/G] \\
\hline 1 & 0.9993 & 39.5344 \\
2 & 0.9994 & 39.3396 \\
5 & 0.9993 & 38.9901 \\
10 & 0.9993 & 38.6321 \\
20 & 0.9993 & 38.9188 \\
50 & 0.9990 & 39.2599 \\
\hline \hline
\end{tabular}




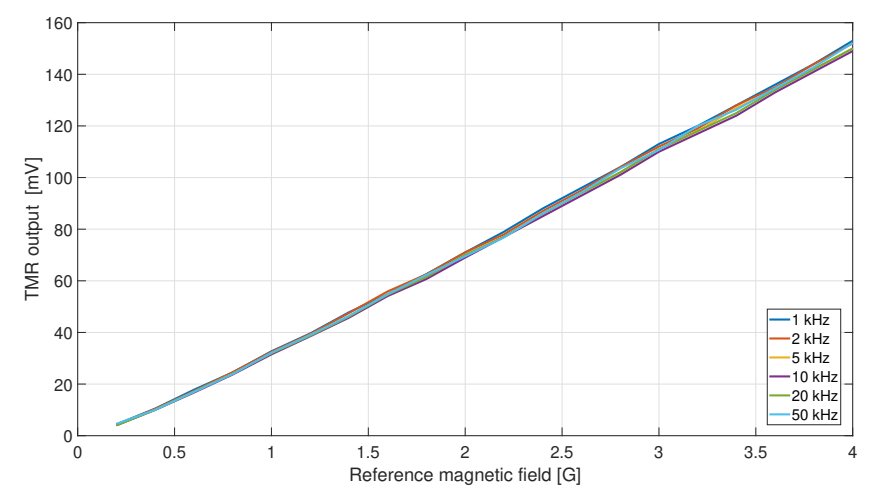

Fig. 7. Input-output relations for the adopted TMR sensor.

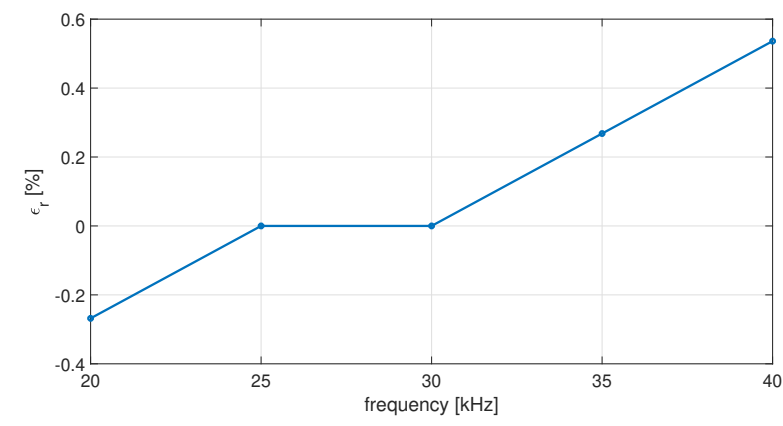

Fig. 8. Frequency stability for the adopted TMR sensor.
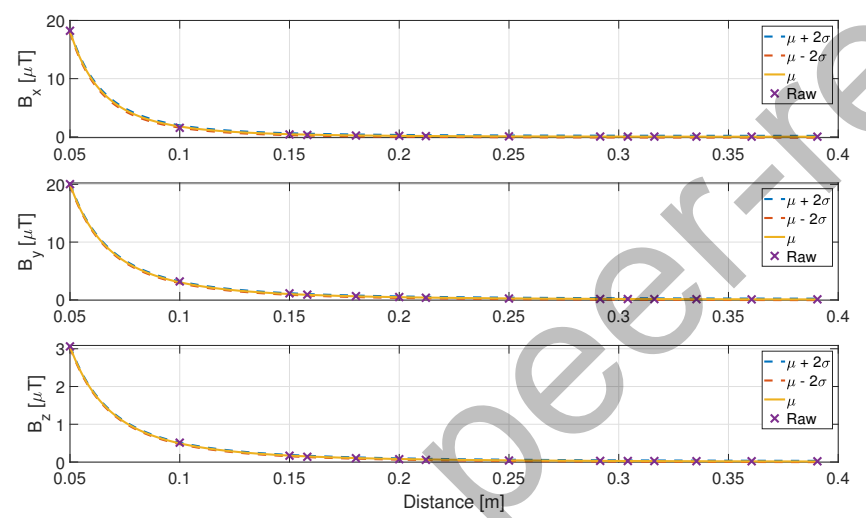

Fig. 9. Relationship of the measured field as a function of distance.

A final test was performed, in which the behavior of the TMR along the three axes was analyzed versus the distance between the sensor and a transmitter coil. The transmitting frequency has been set to $40 \mathrm{kHz}$ in this experiment. In particular, in Fig. 9, raw measurement data (TMR output normalized by its sensitivity), reported with a " $x$ " in the plot, are fitted with a power law with one coefficient (model $a x^{b}$ ). The fitted curve is reported as $\mu$ in each subfigure along with its $95 \%$ boundaries (named as $\mu-2 \sigma, \mu+2 \sigma$ ). The obtained values (pairs [a,b]) are: [0.08e-8, -3.35], [0.57e-8, -2.72], [0.10e-8, -2.63], for $x, y$ and $z$ axes, respectively. The obtained fitting curves have reported $R^{2}$ values greater than 0.999. Such result has confirmed how the measured field follow a distance relation in accordance with the theoretical model.

\section{B. Obtained performance for both systems}

In order to compare the performance of the two systems, six points were considered within the domain, chosen to test the systems in points with different coordinates in all directions. The coordinates of the analyzed points are shown in Table III according to the Cartesian Reference System depicted in Figs. $1,2$.

TABLE III

COORDINATES OF THE EXAMINED POINTS.

\begin{tabular}{cccc}
\hline \hline Positions & $\mathbf{x}[\mathbf{m m}]$ & $\mathbf{y}[\mathbf{m m}]$ & $\mathbf{z}[\mathbf{m m}]$ \\
\hline 1 & 75 & 225 & 20 \\
2 & 150 & 75 & 20 \\
3 & 150 & 150 & 170 \\
4 & 75 & 150 & 170 \\
5 & 150 & 225 & 20 \\
6 & 225 & 225 & 20 \\
\hline \hline
\end{tabular}

In order to give a statistical value to the tests, $\mathrm{N}$ repeated measures were considered for each examined point. After obtaining an estimate of the unknown position, as described in section II, the average positioning error (5) is determined in the three spatial directions respect to the real position. In (5) $\epsilon_{x, i}$, $\epsilon_{y, i}$ and $\epsilon_{z, i}$ represent the committed errors, by single measure, on the $x, y, z$ coordinates respectively. In particular, $\mathrm{N}$ was chosen equal to 10 . Fig. 10 shows the obtained performances for the examined positions, for both systems. The vertical lines on each bar represent the experimental standard deviations. It can be noted that in some cases (positions 1 and 2) the obtained performances with both systems are compatible, while in the other cases with the coil system the obtained errors are lower than in the sensor system.

$$
\epsilon=\frac{1}{N} \sum_{i=1}^{N} \frac{\epsilon_{x, i}+\epsilon_{y, i}+\epsilon_{z, i}}{3}
$$

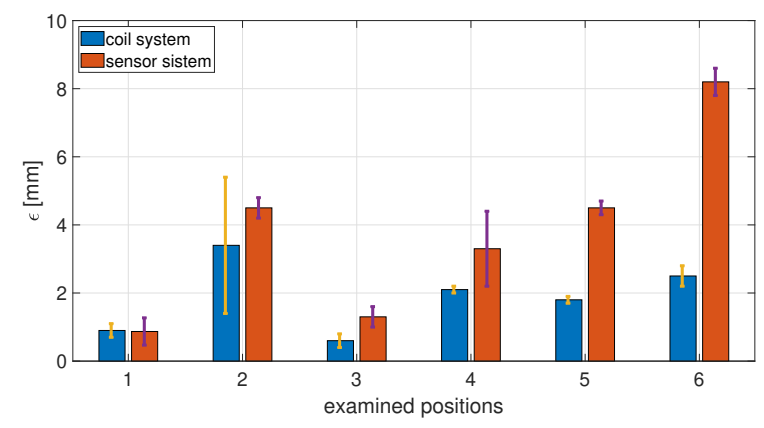

Fig. 10. Obtained positioning errors for both systems. 


\section{CONCLUSIONS}

The aim of the paper has been the usage of a TMR triaxial sensor as a receiving device, in a first prototype of a short-range localization system based on magnetic field measurements. As first task, a preliminary sensor characterization was carried out to verify the reliability of the measurements. Secondly, the performance of the sensor system was evaluated in comparison with that of another magnetic localization system with a different architecture (coil system). The obtained results showed that, although the performance of the sensor system is worse than those of the coil system, the errors committed are anyway under $9 \mathrm{~mm}$. This allows to investigate and improve some aspects of the system design in order to improve the performance. In particular, a new arrangement of the three sensors used to reduce the distances between them and obtain a better alignment, an optimization of the metrological characteristics of the sensors and a suitable procedure to optimally choose the TMR sensor power supply value will be faced as future developments to reduce the localization errors and make the system suitable for submillimeter accuracy requiring applications.

\section{ACKNOWLEDGMENTS}

This research was financially supported by the Italian Ministry of Education, University and Research (MIUR) through the grant PRIN 2015 (Projects of Relevant National Interest, project code: 2015C37B25) and partially funded by the Dipartimenti di Eccellenza, Italian Ministry of Education, University and Research funding programme.

\section{REFERENCES}

[1] E. Strömmer, M. Hillukkala, and A. Ylisaukko-oja, "Ultra-low power sensors with near field communication for mobile applications," in Wireless Sensor and Actor Networks, L. Orozco-Barbosa, T. Olivares, R. Casado, and A. Bermúdez, Eds. Boston, MA: Springer US, 2007, pp. 131-142.

[2] Q. Zhu, R. Wang, Q. Chen, Y. Liu, and W. Qin, "Iot gateway: Bridgingwireless sensor networks into internet of things," in 2010 IEEE/IFIP International Conference on Embedded and Ubiquitous Computing, Dec 2010, pp. 347-352.

[3] R. Salpietro, L. Bedogni, M. Di Felice, and L. Bononi, "Park here! a smart parking system based on smartphones' embedded sensors and short range communication technologies," in 2015 IEEE 2nd World Forum on Internet of Things (WF-IoT), Dec 2015, pp. 18-23.

[4] D. Capriglione, M. Carratù, A. Pietrosanto, and P. Sommella, "Real-time implementation of an ifd scheme for motorcycle sensors," in 2018 IEEE International Instrumentation and Measurement Technology Conference (I2MTC), May 2018, pp. 1-6.

[5] — " "Online fault detection of rear stroke suspension sensor in motorcycle," IEEE Transactions on Instrumentation and Measurement, vol. 68, no. 5, pp. 1362-1372, May 2019.

[6] L. Angrisani, P. Arpaia, F. Bonavolonta, and R. S. Lo Moriello, "Academic fablabs for industry 4.0: Experience at university of naples federico ii," IEEE Instrumentation Measurement Magazine, vol. 21, no. 1, pp. 6-13, February 2018.

[8] L. Angrisani, F. Bonavolontà, G. Cavallo, A. Liccardo, and R. S. L. Moriello, "On the measurement uncertainties of thz imaging systems based on compressive sampling," Measurement, vol. 116, pp. 83 - 95, 2018. [Online]. Available: http://www.sciencedirect.com/science/article/pii/S0263224117306541
[7] M. Carratù, A. Pietrosanto, P. Sommella, and V. Paciello, "Semi-active suspension system for motorcycles: From the idea to the industrial product," in 2018 IEEE International Instrumentation and Measurement Technology Conference (I2MTC), May 2018, pp. 1-6.

[9] L. Angrisani, F. Bonavolontà, R. S. L. Moriello, A. Andreone, R. Casini, G. Papari, and D. Accardo, "First steps towards an innovative compressive sampling based-thz imaging system for early crack detection on aereospace plates," in 2014 IEEE Metrology for Aerospace (MetroAeroSpace), May 2014, pp. 488-493.

[10] A. Bernieri, L. Ferrigno, M. Laracca, and A. Rasile, "Eddy current testing probe based on double-coil excitation and gmr sensor," IEEE Transactions on Instrumentation and Measurement, vol. 68, no. 5, pp. 1533-1542, May 2019.

[11] G. Cerro, L. Ferrigno, M. Laracca, F. Milano, P. Carbone, A. Comuniello, A. D. Angelis, and A. Moschitta, "An accurate localization system for nondestructive testing based on magnetic measurements in quasiplanar domain," Measurement, vol. 139, pp. 467 - 474, 2019.

[12] E. Rovini, C. Maremmani, and F. Cavallo, "How wearable sensors can support Parkinson's disease diagnosis and treatment: a systematic review," Frontiers in neuroscience, vol. 11, p. 555, 2017.

[13] F. Santoni, A. De Angelis, I. Skog, A. Moschitta, and P. Carbone, "Calibration and characterization of a magnetic positioning system using a robotic arm," IEEE Transactions on Instrumentation and Measurement, vol. 68, no. 5, pp. 1494-1502, May 2019.

[14] V. Pasku, A. De Angelis, M. Dionigi, G. De Angelis, A. Moschitta, and P. Carbone, "A positioning system based on low-frequency magnetic fields," IEEE Transactions on Industrial Electronics, vol. 63, no. 4, pp. 2457-2468, April 2016

[15] G. De Angelis, V. Pasku, A. De Angelis, M. Dionigi, M. Mongiardo, A. Moschitta, and P. Carbone, "An indoor ac magnetic positioning system," IEEE Transactions on Instrumentation and Measurement, vol. 64, no. 5, pp. 1267-1275, May 2015.

[16] A. Bernieri, G. Betta, L. Ferrigno, M. Laracca, and A. Rasile, "Characterization of a tmr sensor for ec-ndt applications," in Convegno Nazionale Sensori. Springer, 2018, pp. 229-236.

[17] G. Cerro, L. Ferrigno, M. Laracca, F. Milano, P. Carbone, A. Comuniello, A. De Angelis, and A. Moschitta, "Probe localization by magnetic measurements in eddy-current nondestructive testing environment," in 2018 5th IEEE International Workshop on Metrology for AeroSpace (MetroAeroSpace), June 2018, pp. 45-49.

[18] P. Bellitti, M. Bona, M. Borghetti, E. Sardini, and M. Serpelloni, "Application of a modular wearable system to track workers' fingers movement in industrial environments," in 2019 II Workshop on Metrology for Industry 4.0 and IoT (MetroInd4.0 IoT), June 2019, pp. 137-142.

[19] A. Bodini, E. Cantu', M. Serpelloni, E. Sardini, and S. Tonello, "Design and implementation of a microsensor platform for protein detection realized via 3-d printing," in 2018 IEEE Sensors Applications Symposium (SAS), March 2018, pp. 1-6.

[20] L. Angrisani, D. Capriglione, G. Cerro, L. Ferrigno, and G. Miele, "On employing a savitzky-golay filtering stage to improve performance of spectrum sensing in CR applications concerning vdsa approach," Metrology and Measurement Systems, vol. 23, no. 2, pp. 295-308, 2016.

[21] G. Betta, D. Capriglione, G. Cerro, L. Ferrigno, and G. Miele, "The effectiveness of savitzky-golay smoothing method for spectrum sensing in cognitive radios," in 2015 XVIII AISEM Annual Conference, Feb 2015, pp. $1-4$.

[22] L. Angrisani, D. Capriglione, G. Cerro, L. Ferrigno, and G. Miele, "Optimization and experimental characterization of novel measurement methods for wide-band spectrum sensing in cognitive radio applications," Measurement, vol. 94, pp. 585 - 601, 2016.

[23] J. C. Lagarias, J. A. Reeds, M. H. Wright, and P. E. Wright, "Convergence properties of the nelder-mead simplex method in low dimensions," SIAM Journal on optimization, vol. 9, no. 1, pp. 112-147, 1998.

[24] F. Reverter and O. Casas, "Interfacing differential resistive sensors to microcontrollers: A direct approach," IEEE Transactions on Instrumentation and Measurement, vol. 58, no. 10, pp. 3405-3410, Oct 2009. 